\title{
Enhanced surveillance for childhood hepatitis B virus infection in Canada, 1999-2003
}

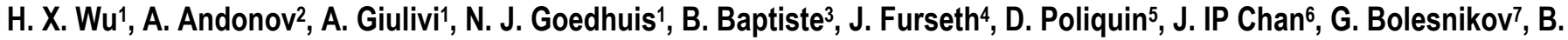 \\ Moffat $^{8}$, S. Paton ${ }^{1}$, and J. Wu ${ }^{1}$
}

1 Blood borne Pathogens Section, Blood Safety Surveillance and Health Care Acquired Infection Division, Centre for Infectious Disease Prevention and Control, Public Health Agency of Canada, Ottawa, ON, Canada

2 National Microbiology Laboratory, Canadian Science Centre for Human and Animal Health, Public Health Agency of Canada, Winnipeg, MB, Canada

3 Capital Health, Edmonton, AB, Canada

4 Calgary Health Region, Calgary, AB, Canada

5 City of Ottawa, Public Health and Long Term Care Branch, Ottawa, ON, Canada

6 Vancouver Coastal Health, Vancouver, BC, Canada

7 Department of Health and Wellness, NB, Canada

8 British Columbia Centre for Disease Control (BCCDC), BC, Canada

Corresponding address: Hong-Xing Wu, MD, PhD, 0601E2, Health Canada Buildings No. 6, Tunney's Pasture, Ottawa, Ontario, Canada K1A 0L2. Tel: +1 (613) 957-3047 Fax:+1 (613) 952-6668 e-mail: hong-xing_wu@hc-sc.gc.ca

Received: 2005.08.19; Accepted: 2005.09.06; Published: 2005.10.01

Since hepatitis B virus (HBV) infection can have serious sequelae, especially if infection occurs during childhood, there is a continuing need to examine its epidemiology so as to inform control measures. We analyzed trends in disease incidence and patterns of hepatitis B virus (HBV) transmission in both Canadian-born and non-Canadian-born children from 1999 to 2003, through the Enhanced Hepatitis Strain Surveillance System. Amongst Canadian-born children, the incidence of newly identified HBV infection per 100,000 declined significantly during the study period from 1.4 in 1999, to 0.5 in 2003 (RR, 0.75 per year; 95\% CI, 0.60-0.95). Amongst non-Canadian-born children, the incidence of HBV infection per 100,000 ranged from 9.4 to 16.3, during the study period (linear trend test, $\mathrm{p}=0.69$ ). Poisson regression analysis revealed that non-Canadian-born children were more likely to have HBV infection (RR, $12.3 ; 95 \% \mathrm{Cl}, 7.6$ to 19.8), than Canadian-born children. HBV infection was found to be more common among children emigrating from high endemic area, than among Canadian-born children. Current Canadian immunization policy should take into consideration the protection of all children against HBV infection, including those coming from countries where mass hepatitis B vaccination programs have still not been launched.

Key words: incidence, hepatitis $B$, surveillance, children, birthplace

\section{INTRODUCTION}

In certain parts of the world, Hepatitis B $(\mathrm{HB})$ is a significant cause of chronic liver disease. It is estimated that more than 300 million adults and children worldwide are chronically infected with hepatitis B virus (HBV) [1]. Infections acquired in childhood are responsible for the largest majority of chronic HBV infection, with its attendant complications of cirrhosis and hepatocellular carcinoma [2]. Preventing HBV infection in young children is therefore of particular importance.

There is a small amount of literature available on the prevalence of HBV infection in the general Canadian population. In previous studies of selected populations in Canada, HBV surface antigen ( $\mathrm{HBsAg}$ ) seroprevalence rates were estimated to be between $0.24 \%$ and $0.47 \%$ in people aged 14-30 years from a northern Ontario town [3]. These rates were estimated to be $5-15 \%$ in adults from Southeast Asia [4], and $0.1-0.5 \%$ in Canadian first-time blood donors [5]. In a 1995 survey of 1200 school children aged 8-10 years in Quebec, none were found to be positive for HBsAg, or the antibody to the HBV core antigen [6]. Since the early 1990s, an increasing number of immigrants from high and intermediate endemic areas have entered Canada [7]. This immigration may be changing the distribution of HBV infection in Canada.

Since 1997, all provinces and territories in Canada have launched universal school-based vaccination programs in order to prevent HBV infection in the early years of life [8]. There is a continuing need to examine the effectiveness of the Canadian HBV vaccination strategy. Regarding the possible need for new HBV prevention strategy in Canada, the present study was therefore undertaken to analyze national Enhanced Hepatitis Strain Surveillance System (EHSSS) data collected from 1999 through 2003.

\section{METHODS}

\section{Population and measures}

The detailed surveillance methods used in the EHSSS have been described elsewhere [9]. From 1999 to 2003, the number of health regions participating in the EHSSS has increased greatly; in 2003, 7 public health jurisdictions were participating. These health regions were distributed throughout the country, covering approximately $25 \%$ of the Canadian population (7.7 / 31 million total population), including approximately one third of all new immigrants to the country. Children with HBV infection, diagnosed between January 1, 1999 and December 31, 2003, were included in the analysis. For the purpose of this analysis, a child was defined as a person younger than 16 years of age. This was calculated using the date for first positive test for HBsAg minus the date of birth. A newly identified case is a person who has a laboratory confirmed diagnosis of HBV infection for the first time. The diagnosis 
of HBV infection was based on positive HBsAg. The case definition for confirmed acute HBV infection includes the presence of HBsAg combined with IgM antibody to the HBV core (anti-HBc). A child was defined as Canadianborn if the child's birthplace was reported to be in Canada, or if the date of the mother's immigration to Canada preceded the child's date of birth. A person who did not meet these criteria was classified as non-Canadian-born.

Virological laboratories, which were performing confirmatory testing for $\mathrm{HB}$ in each jurisdiction, either automatically reported or were contacted regularly for newly identified $\mathrm{HB}$ cases, laboratory test results, and other related information. A standardized data collection form was used. Information was obtained from basic demographic characteristics, diagnostic test results, presenting clinical syndromes, and basic epidemiologic information, including potential risk factors. Questionnaire data from all seven-health regions were entered into a longitudinal database for storage and analysis. Efforts were made to check the data records for accuracy and to eliminate any duplication. Audits were conducted at each 3-month interval during the study period in order to evaluate completeness of reporting, and to capture any cases that were not initially identified. Missing values and irregularities were reassessed with the help of investigators at each health region. Programmed computer checks and cross-tabulations are used to reduce the risk of typing errors.

\section{Statistical methods}

SAS 8.2 statistical programs were used for analysis. The annual population estimates from the 2001 Canadian Census for birthplace classifications were used to calculate rates for newly identified HB cases in both Canadian-born and non-Canadian-born children [7]. Poisson regression analysis was used to compare the rates of the reported disease over time using: gender, calendar year, age and birthplace, which were the only variables available for denominator data. After examining the full model, we constructed the final model using a stepwise procedure. All possible interactions were considered, provided that a hierarchical model was maintained. A common reason for poorly fitting Poisson models is overdispersion of counts, which can result in overstating the statistical significance of differences between time periods [10]. To protect against this, significance levels were computed using a quasilikelihood estimation of the Poisson model, which included a dispersion parameter. To assess the adequacy of our models, we computed goodness-of-fit statistics on the residual deviance. Differences in proportions were determined by a chi-square test and, where necessary, Fisher's exact test. The Kruskal-Wallis test was used to compare the ranked distribution of ordinal variables. Twotailed $p$ values were reported where $P<0.05$ was considered statistically significant.

\section{RESULTS}

From 1998 to 2003, a total of 197 newly diagnosed HB cases in children were reported to the EHSSS. Of these, 2 clinically recognized HB cases with neither HBsAg nor IgM anti-HBc results were excluded. Thus, $195 \mathrm{HB}$ cases in children were included in the final analysis. Of these children, 36 (18.5\%) were Canadian-born, 156 (80.0\%) were non-Canadian-born, and for $3(1.5 \%)$ the birthplace was not reported. The 156 non-Canadian-born children came primarily from Asia (76.3\%), with 19.9\% from Africa, and
$3.8 \%$ from other areas. In all but four of the 195 children the infection was asymptomatic; the clinical characters of nonCanadian-born children were similar with those of Canadian-born children. Nine out of the 195 children were identified as being infected with acute HBV. No significant statistical difference in the proportion of children with acute HBV infection was found between Canadian-born and non-Canadian-born children (Fisher's exact test, $p>0.2)$. The infection was diagnosed significantly earlier in Canadian-born children (median age 4 years) compared to non-Canadian-children (median age 13 years) (Kruskal Wallis test, $\mathrm{p}<0.01$ ). Eight out of 75 possessed an aniline aminotransferase (ALT) level $>2$ times the upper reference value of Canada (i.e., > $98 \mathrm{IU} / \mathrm{L}$ ). The proportion of children with abnormal ALT did not reveal a significant difference between Canadian-born and non-Canadian-born children, at the time of diagnosis (Fisher's exact test, $\mathrm{p}>0.1$ ). In non-Canadian-born children, the mode of transmission was determined to be vertical in $17.3 \%$, horizontal in $43.0 \%$, and unknown in $37.7 \%$. In the Canadian-born subgroup, horizontal transmission and neonatal maternal transmission were frequent $(27.8 \%$, and $63.9 \%$, respectively), although in $8.3 \%$ of the cases the cause of infection was unknown.

Table 1 illustrates the variation in rates of newly identified HBV infection through birthplace, age, gender, and year of reporting. Year-to-year trends in the rate of HBV infection in both Canadian-born and non-Canadianborn children are shown in Fig. 1. Amongst Canadian-born children, the rate of newly identified HBV infection declined from 1.4 per 100,000 in 1999 , to 0.5 per 100,000 in 2003. This was statistically significant, with an estimated rate ratio (RR) for successive years of 0.75 [95\% confidence interval (CI), 0.60-0.95; $\mathrm{p}=0.017]$. Amongst non-Canadianborn children, the rate of HBV infection per 100,000 ranged from 9.4 to 16.3 in the study period (test for linear trend, $p=0.69$ ).

The final Poisson regression model included main effects for birthplace, age group, calendar year, an interaction term between age group and birthplace, and an interaction term between calendar year and birthplace on the RR scale. That is, the effect of both age and calendar year depended on a child's birthplace. Gender was not retained, for its presence in the model did not affect the other coefficients. Table 2 illustrates rate ratios estimated from the model. After adjustment, the rate of $\mathrm{HBV}$ infection in non-Canadian-born children was 12.3 times higher than in Canadian-born children (95\% CI 7.6-19.8). Among non-Canadian-born children aged 0-4 and 5-9 years, the risk of acquiring $\mathrm{HB}$, and being newly identified, was significantly lower than among those aged 10 to 15 years (RR 0.18, 95\% CI 0.11-0.28 and RR 0.17, 95\% CI 0.110.28 , respectively). In contrast, compared with the baseline rate in Canadian-born children aged 10 to 15 years, Canadian-born children aged 0 to 4 years had a 3 -fold greater risk of newly acquiring $\mathrm{HBV}$ infection (RR 3.56, 95\% CI 1.57-8.09).

\section{DISCUSSION}

Within those health regions covered by the EHSSS, the Public Health Agency of Canada supports staff in conducting enhanced surveillance, intensive case investigations, and serologic follow-up. Therefore, it is reasonable to presume that the present surveillance captured most of the newly identified, clinically recognized 
cases with HBV infection in children. Furthermore, the present surveillance was carried out using a predefined operating protocol and predefined questionnaires for all seven health regions. The notification system was not materially changed during the study period, but augmented throughout by scrutiny of laboratory results and hospital admissions; therefore the incidence trend was not affected. Data collected through the EHSSS would provide valuable evidence of changes in secular trends over reasonably long time periods. They are useful in defining risk groups and monitoring changes in the population.

Over the last 5 years, universal school-based vaccination strategies, as well as the increase in immigration flow and international adoptions, have changed the epidemiology of hepatitis occurring in childhood, in Canada. Our analyses of the EHSSS data collected during 1999-2003, suggest that these factors contribute to a reduced incidence of HBV infections in Canadian-born children, and to an unchanged incidence in non-Canadian-born children. Many non-Canadian born children in our study came from moderately or highly endemic countries, where the majority of the population is infected during childhood either perinatally or through child-to-child transmission [11, 12]. On a national scale, the influx of $\mathrm{HB}$ cases from endemic countries may currently be an important element in HB epidemiology in Canada, and may continue to be so in the foreseeable future. Nevertheless, amongst immigrant groups, improved social status or sociomedical integration, as well as the possibility of vaccination, particularly among children, could reduce the risk of household spread [13]. Given the strong association between place of birth, and the rate of reported $\mathrm{HBV}$ infection in children, it is suggested that focused screening for HBsAg in non-Canadian-born children may provide the means for detecting most HBsAg carriers with heretofore undiagnosed HB. Screening in these children for HBV is important for identifying those at risk of developing long-term consequences of chronic HBV infection, and for developing opportunities to vaccinate susceptible contacts to prevent further transmission [2].

One of the most striking results, occurring during the 1999 to 2003 period, was the incidence of newly identified HBV infection, which decreased significantly in Canadianborn children, particularly among children aged 10-15 years. This trend of decrease is consistent with the results of an epidemiological survey in British Columbia, Canada, which demonstrates a significant decrease in the incidence of acute HBV infection in people aged 15 to 24 years [13]. A decrease in the incidence, observed in Canadian-born children, can be a result of the general improvement in the standard of living, hygiene, and the introduction of public health measures, such as refinement in blood screening, the use of universal precautions in a medical setting, and the implementation of universal school-based vaccination programs [8]. The incidence of acute HB in the general Canadian population has not increased, despite a growing number of chronic HBV-carriers [14, 15]. The risk of transmission may increase when the children with chronic HBV infection reach adulthood and establish sexual contacts. The analysis of our surveillance data supports the need for continuing universal childhood immunization in an attempt to prevent chronic $\mathrm{HB}$ and the associated substantial burden of HB-related chronic liver disease.
The HBV screening and immunization guidelines have existed in Canada since the early 1990s to prevent perinatal transmission [8]. Regarding route of transmission, it is still noteworthy that a relatively high proportion of the newly identified HBV infection in Canadian-born children was attributable to perinatal transmission from a mother with HBV infection during the past five years. It is presumed that this is due to the recommended antenatal screening of pregnant women from risk groups, and the subsequent immunization of their newborns [16]. Infection in Canadian-born children seems to be primarily acquired through vertical transmission from the mother. This is in agreement with findings from other studies dealing with low endemic areas [17]. It is anticipated that the identification of babies at high risk for perinatal HBV infection will be more complete, and the administration of immunoprophylaxis at birth will become more routine. In data from the EHSSS, we found a relatively high rate of vertical and horizontal transmission in non-Canadian born children. This is consistent with the fact that most of the children came from highly endemic countries where vertical and horizontal transmission is the major route of virus acquisition [11, 18]. Cases amongst non-Canadianborn children were on average older than cases amongst Canadian-born children $(p<0.01)$. This could be due partly to the delay between infection and diagnosis in nonCanadian-born children, although it would be interpreted that the age of acquiring HBV infection between the two groups of children may exist differently. In this study, it was not possible to estimate the number of HBV infections acquired in early life. Neither was it possible to estimate the number of children from this study who may have acquired infections occurring among them after they arrived in Canada, as the study was not designed to look at these factors.

In our analyses, there are a number of limitations and potential sources of bias that may influence results, and therefore merit discussion. First, all of the analyses performed were based on the aggregate dataset from the EHSSS; thus the reported cases are likely to represent an underestimate of the true incidence of infection. This is due to the majority of childhood HB cases being asymptomatic. While these factors may affect the yearly incidence estimate, changes in the incidence rate would be reliable as long as the proportion of asymptomatic cases remained constant. We focused the analysis on all newly identified $\mathrm{HB}$ cases in children; therefore data collected through the EHSSS would provide valuable evidence of changes in secular trends over reasonably long time periods. Second, the ascertainment of infection would have been better among non-Canadian-born children. It is possible that immigrant family members were more likely to come to the attention of the reporting system, if patients were tested. The extent of ascertainment bias is likely to be minimal, for, as we know, Canada has not established a screening program for new immigrants. The study of our case series did not reveal differences in the severity of HBV disease between the two groups of children. Third, due to new immigrant migration to Canada from high endemic areas in recent years, there exists the expectation of a net increase in $\mathrm{HB}$ cases in children, generating a conservative bias. Fourth, only some cities (Vancouver, Edmonton, Calgary) with significant immigrant population, but not Montreal and Toronto, are part of this sentinel surveillance system. However, the EHSSS covers jurisdictions from coast to 
coast spanning Western Canada, the Prairies, Central Canada, and the Atlantic region, involving Englishspeaking, French-speaking and other diverse ethnic populations. These regions cover a quarter of the Canadian population.

In conclusion, to prevent new HBV infections, it is important to ensure the screening of pregnant women, and the vaccination of children born to HBsAg-positive mothers. In addition, the immigrant population should be targeted for screening, education, and vaccination in order to reduce $\mathrm{HBV}$ transmission. Continued surveillance of HBV infection among children is necessary to implement future immunization strategies.

\section{Conflict of interest} exists.

The authors have declared that no conflict of interest

\section{ACKNOWLEDGMENT}

The authors would like to acknowledge the contributions of Gregory Zaniewski and Zhiyong Hong for their epidemiologic expertise, Marina Kanabe for administrative support, Qiong $\mathrm{Li}$ and Lisa Sockett for technical support, and Nick Karitsiotis for assisting in the development of the Enhanced Hepatitis Strain Surveillance System.

\section{REFERENCES}

1. Lai CL, Ratziu V, Yuen MF, Poynard T. Viral hepatitis B. Lancet 2003; 362: 2089-2094.

2. Broderick AL, Jonas MM. Hepatitis B in children. Semin Liver Dis 2003; 23: 59-68.

3. Glasgow KW, Schabas R, Williams DC, Wallace E, Nalezyty LA. A population-based hepatitis B seroprevalence and risk factor study in a northern Ontario town. Can J Public Health 1997; 88: 87-90

4. Wong WW, Minuk GY. A cross-sectional seroepidemiologic survey of chronic hepatitis B virus infections in Southeast Asian immigrants residing in a Canadian urban centre. Clin Invest Med 1994; 17: 443-447

5. Chiavetta JA, Escobar M, Newman A, et al. Incidence and estimated rates of residual risk for HIV, hepatitis C, hepatitis B and human Tcell lymphotropic viruses in blood donors in Canada, 1990-2000. CMAJ 2003; 169: 767-773.

6. Duval B, Boulianne N, De Serres G, et al. Should children with isolated anti-HBs or anti-HBc be immunized against hepatitis $B$ virus?[letter]. JAMA 1997; 278: 1064.

7. [Internet] Statistics Canada. Selected places of birth for the immigrant population, for Canada, provinces, territories, census metropolitan areas and census agglomerations, 1996 and 2001. Accessed October 2004. http:// www12.statcan.ca/english/census01/home/index.cfm

8. Tepper ML, Gully PR. Hepatitis B. CMAJ 1997, 156: 1033-1034.

9. Zou S, Zhang J, Tepper M, Giulivi A, et al. Enhanced surveillance of acute hepatitis B and C in four regions in Canada, 1998 to 1999. Can J Infect Dis 2001; 12: 357-363.

10. Rothman KJ, Greenland S. Modern Epidemiology, $2^{\text {nd }}$ ed. Philadephia, PA: Lippincott Williams \& Wilkins Press. 1998: 404-432.

11. Chen $\mathrm{CH}$, Chen YY, Chen GH, et al. Hepatitis B virus transmission and hepatocarcinogenesis: a 9 year retrospective cohort of 13676 relatives with hepatocellular carcinoma. J Hepatol 2004; 40: 653-659

12. Hurie MB, Mast EE, Davis JP. Horizontal transmission of hepatitis B virus infection to United States-born children of Hmong refugees. Pediatrics 1992; 89: 269-673.

13. Patrick DM, Bigham $\mathrm{M}, \mathrm{Ng} \mathrm{H}$, et al. Elimination of acute hepatitis B among adolescents after one decade of an immunization program targeting Grade 6 students. Pediatr Infect Dis J 2003; 22: 874-877.

14. Zhang J, Zou S, Giulivi A. Epidemiology of hepatitis B in Canada. Can J Infect Dis 2001; 12: 345-350

15. Wu HX, Wu J, Zaniewski G, Goedhuis N, Giulivi A. Enhanced sentinel surveillance of hepatitis B in Canada, 1998-2003: Implication for immunization policy. Can J Infect Dis \& Med Microbiol 2004; 15: 359.
16. Okun NB, Larke RPB, Waters JR, Joffres MR. Success of a program of routine prenatal screening for hepatitis $B$ surface antigen: the first 2 years. CMAJ 1990; 143: 1317-1321.

17. Ordog K, Szendroi A, Szarka K, et al. Perinatal and intrafamily transmission of hepatitis $\mathrm{B}$ virus in three generations of a lowprevalence population. J Med Virol 2003; 70: 294-204

18. Kashiwagi S, Hayashi J, Nomura $\mathrm{H}$, at al. Changing pattern of intrafamilial transmission of hepatitis B virus in Okinawa, Japan. Am J Epidemiol 1988; 127: 783-787

\section{Tables}

Table 1. Rate ratio of newly identified HBV infection in children, EHSSS, 1999-2003

\begin{tabular}{|c|c|c|c|}
\hline Variable & $\begin{array}{c}\text { Incidence rate } \\
/ 100000\end{array}$ & $\begin{array}{c}\text { Rate } \\
\text { ratio }\end{array}$ & $\begin{array}{c}95 \% \text { confidence } \\
\text { interval }\end{array}$ \\
\hline Origins & & & \\
\hline Non-Canadian born & 13.80 & 16.23 & 8.36 to 31.53 \\
\hline Canadian born & 0.85 & $1^{\#}$ & \\
\hline Year & & & \\
\hline 1999 & 5.24 & 1.94 & 0.51 to 7.40 \\
\hline 2000 & 4.34 & 1.60 & 0.43 to 5.90 \\
\hline 2001 & 4.80 & 1.77 & 0.50 to 6.24 \\
\hline 2002 & 2.53 & 0.93 & 0.23 to 3.72 \\
\hline 2003 & 2.71 & $1^{\#}$ & \\
\hline Gender & & & \\
\hline Male & 3.94 & 1.19 & 0.51 to 2.75 \\
\hline Female & 3.32 & $1^{\#}$ & \\
\hline Age (years) & & & \\
\hline 0 to 4 & 2.71 & $1^{\#}$ & \\
\hline 5 to 9 & 1.56 & 0.58 & 0.15 to 2.27 \\
\hline 10 to 15 & 6.06 & 2.24 & 0.84 to 5.97 \\
\hline
\end{tabular}

"Baseline group

Table 2. The incidence rate ratios computed with multivariate analysis by Poisson regression*

\begin{tabular}{|c|c|c|}
\hline Characters & $\begin{array}{l}\text { Rate } \\
\text { ratio }\end{array}$ & $\begin{array}{c}95 \% \text { confidence } \\
\text { interval for rate ratio }\end{array}$ \\
\hline Non-Canadian vs Canadian-born & 12.27 & 7.60 to 19.81 \\
\hline $\begin{array}{l}\text { Calendar year for non-Canadian-born } \\
\text { children }\end{array}$ & 0.98 & 0.87 to 1.10 \\
\hline Calendar year for Canadian-born children & 0.75 & 0.60 to 0.95 \\
\hline $\begin{array}{c}0 \text { to } 4 \text { years vs } 10-15 \text { years for Canadian- } \\
\text { born children }\end{array}$ & 3.56 & 1.57 to 8.09 \\
\hline $\begin{array}{c}5 \text { to } 9 \text { years vs } 10-15 \text { years for Canadian- } \\
\text { born children }\end{array}$ & 1.10 & 0.40 to 3.04 \\
\hline $\begin{array}{l}0 \text { to } 4 \text { years vs } 10-15 \text { years for non- } \\
\text { Canadian-born children }\end{array}$ & 0.18 & 0.11 to 0.28 \\
\hline $\begin{array}{l}5 \text { to } 9 \text { years vs } 10-15 \text { years for non- } \\
\text { Canadian-born children }\end{array}$ & 0.17 & 0.11 to 0.28 \\
\hline
\end{tabular}

*Poisson regression model adjusted for birthplace, age and calendar year, and interactions between birthplace and age, birthplace and calendar year.

Figure 1. Annual rates of newly identified cases among children with HBV infection<16 years old, 1999 - 2003, by birthplace

100

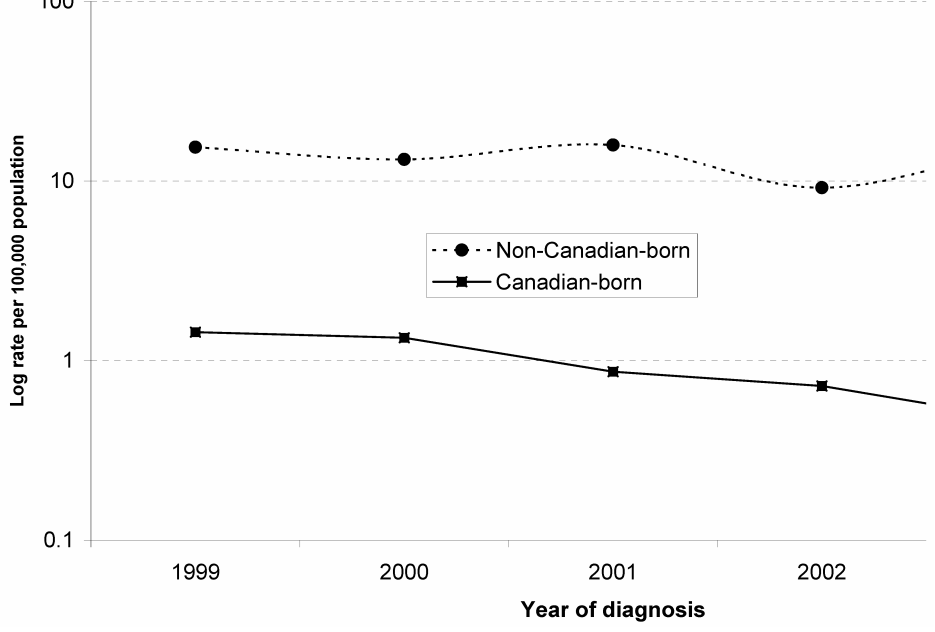

